\title{
PRÉFACE DU TRADUCTEUR
}

Ce livre est le troisième et dernier d'une série de trois recueils d'exercices corrigés traitant des bases de l'analyse réelle. Il s'adresse d'abord aux étudiants, principalement ceux des niveaux L3 et M1, mais les étudiants des niveaux L1 et L2 tireront un grand profit de l'étude du premier chapitre et de la dernière section du second chapitre. Il intéressera aussi les candidats aux concours du CAPES et de l'agrégation de mathématiques qui y trouveront autant les théorèmes qu'ils doivent connaître que des exercices pour les illustrer.

Ce troisième volume traite de l'intégration des fonctions réelles. Le premier chapitre aborde l'intégrale de Riemann et de Riemann-Stieltjes (la dernière section applique ce qui précède aux calculs de volumes, d'aires et de longueurs), le second chapitre s'intéresse à l'intégrale de Lebesgue (la quatrième section porte sur la continuité absolue et la continuité approximative et la dernière section sur les séries Fourier). Chaque section, centrée sur un thème, commence par des exercices relativement simples et se poursuit par des problèmes plus difficiles, certains étant des théorèmes classiques.

Tous les exercices sont corrigés, le plus souvent en détail, ce qui permettra aux étudiants de ne pas «sécher» sur un exercice difficile. Nous les invitons cependant à chercher par eux-mêmes les exercices avant de regarder les solutions et nous insistons aussi sur le fait que les auteurs ne donnent pas nécessairement toutes les étapes d'un calcul lorsqu'ils considèrent que celui-ci ne pose pas de problèmes techniques. C'est bien sur aux étudiants de prendre le temps de rédiger entièrement leurs solutions.

Nous avons ajouter en note les noms de certaines propriétés et relations pour inviter les étudiants à engager des recherches par eux-mêmes. L'index à la fin de l'ouvrage permet de facilement retrouver une définition et la table des renvois permet de voir les liens entre les différents problèmes dans ce volume et dans les deux autres. 
Je tiens à remercier Daniel Guin et Xavier Cottrell pour avoir pris le temps de relire cette traduction et pour les remarques qu'ils m'ont faites afin d'améliorer le style et de corriger les erreurs. Je reste responsable de celles qui subsisteraient. Je souhaite aussi remercier pour sa disponibilité Patrick Fradin, l'auteur du logiciel TeXgraph avec lequel toutes les figures de cet ouvrage et l'illustration de la couverture ont été réalisées.

É. Kouris 